\title{
TRATAMENTO CIRÚRGICO DA DOENÇA PILONIDAL: META-ANÁLISE DOS PRINCIPAIS PROCEDIMENTOS ADOTADOS MUNDIALMENTE
}

\author{
SURGICAL TREATMENT OF PILONIDAL DISEASE: META-ANALYSIS OF THE MAIN \\ PROCEDURES ADOPTED WORLDWIDE
}

\author{
Victor Strassmann, ECBC-SP'; Manoel Carlos Prieto Velhote, ACBC-SP'; \\ Sérgio Santoro ${ }^{3}$; Carlos Eduardo Malzoni, TCBC-SP ${ }^{4}$; Guilherme Tommasi Kappaz ${ }^{5}$
}

\begin{abstract}
RESUMO: Objetivo: Analisar e comparar os diversos procedimentos cirúrgicos descritos para o tratamento da doença pilonidal. Método: Foram selecionados 34 trabalhos publicados em revistas indexadas, totalizando 8698 doentes operados. Realizou-se meta-análise para comparação das sete principais técnicas cirúrgicas descritas na literatura, quanto aos resultados em relação à recidiva e ao tempo de cicatrização no pós-operatório. Resultados: Do total de doentes estudados, houve recidiva em 230 doentes $(2,6 \%)$. O tempo de cicatrização no pós-operatório foi significantemente maior no grupo de excisão sem sutura. As recidivas foram estatisticamente semelhantes nos métodos: excisão sem sutura, marsupialização, incisão e curetagem, excisão e retalho e técnica de Karidakys. Os métodos que apresentaram maior índice de recidiva (estatisticamente significante $-\mathrm{p}<0,001$ ) foram: excisão e sutura primária e o método de Bascom. Conclusão: Conclui-se, por esse estudo, que os resultados em relação à recidiva são estatisticamente semelhantes em todos os métodos, com exceção da excisão e sutura primária e da técnica de Bascom, que apresentaram recidivas mais freqüentes. O tempo de cicatrização foi maior nos indivíduos operados pela técnica de excisão sem sutura primária.
\end{abstract}

Descritores: Cisto pilonidal; Doença pilonidal; Marsupialização; Abscesso sacral; Incisão e curetagem.

\section{INTRODUÇÃO}

A doença pilonidal é uma inflamação crônica dos seios pós-sacrais, e afeta a área de pele posterior ao ânus e recobrindo o sacro, na região da fenda interglútea ${ }^{1}$. Atualmente, entre a maioria dos autores, há consenso em relação à etiologia adquirida da doença, porém o mesmo não ocorre em relação ao melhor tratamento, que ainda é alvo de muitas discussões e incertezas ${ }^{2-4}$.

O tratamento cirúrgico é o preferido pela grande maioria dos autores, e entre os diferentes métodos, a excisão seguida ou não por fechamento da ferida e a incisão do cisto seguida por marsupialização ou curetagem são os principais procedimentos cirúrgicos adotados pelos cirurgiões.

Diversos estudos vêm analisando diferentes modalidades cirúrgicas, mas nenhum deles foi realizado com amostra e controle suficientes para que se pudesse definir o tratamento de escolha para o cisto pilonidal. Com o intuito de esclarecer esta questão, nós realizamos uma metaanálise incluindo a maior parte dos trabalhos da literatura recente, e comparamos os principais procedimentos adotados mundialmente, em relação à recidiva e tempo de cicatrização.

\section{MÉTODO}

Realizou-se um levantamento bibliográfico dos estudos pertencentes à base de dados MEDLINE. Foram selecionados todos os artigos publicados desde 1994 até janeiro de 2002, que incluíssem as diferentes modalidades de tratamento cirúrgico do cisto pilonidal. Mais de $70 \%$ destes artigos estavam disponíveis e foram incluídos na meta-análise. As principais referências bibliográficas citadas nesses artigos que foram publicadas antes de 1994 também foram obtidas e incluídas no estudo. Os artigos que não estavam disponíveis ou que não analisaram as taxas de recidiva dos procedimentos foram excluídos deste estudo.

Devido à existência de muitas modalidades diferentes, os procedimentos cirúrgicos foram agrupados em sete categorias de acordo com as suas características. As sete categorias são: excisão sem sutura primária (ferida aberta), excisão com sutura primária (ferida fechada), marsupialização, incisão seguida de curetagem, uso de retalhos (incluindo todos os tipos de procedimentos com retalhos), procedimento de Bascom e procedimento de Karidakys. Os resultados de cada estudo foram agrupados, e calculou-se o tempo médio de cicatrização e taxas de recidiva. As taxas de recidiva foram comparadas estatísticamente através do teste de Mantel-Haenszel.

1. Doutor em Medicina pelo Departamento de Cirurgia da Faculdade de Medicina da Universidade de São Paulo; Professor Assistente da Disciplina de Cirurgia do Aparelho Digestivo da Faculdade de Medicina da Universidade de São Paulo; Fellow do American College of Surgeons.

2. Professor Assistente da Disciplina de Cirurgia Pediátrica da Faculdade de Medicina da Universidade de São Paulo.

3. Mestre em Cirurgia pelo Departamento de Gastroenterologia da Faculdade de Medicina da Universidade de São Paulo.

4. Mestre em Cirurgia pelo Departamento de Gastroenterologia da Faculdade de Medicina da Universidade de São Paulo; Fellow do American College of Surgeons

5. Acadêmico de Medicina da Faculdade de Medicina do ABC, Santo André (SP)

Recebido em 07/10/2003

Aceito para publicação em 08/06/2004

Trabalho realizado no Hospital Israelita "Albert Einstein", São Paulo (SP) e apresentado no "The 13" Annual Colorectal Disease Symposium" Cleveland Clinic Florida, Fort Lauderdale, Florida (EUA), 16 de fevereiro de 2002. 


\section{RESULTADOS}

A meta-análise incluiu 34 estudos. A Tabela 1 lista as publicações e dados relacionados a cada estudo. Os artigos que contemplavam mais de um procedimento cirúrgico foram divididos de acordo com o procedimento, totalizando 44 itens na Tabela 1 .

Tabela 1 - Lista de todos os artigos incluídos na meta-análise.

\begin{tabular}{|c|c|c|c|c|c|c|c|}
\hline Autor & Ano & Método & $\begin{array}{l}\text { Número } \\
\text { de Casos }\end{array}$ & $\begin{array}{l}\text { Número de } \\
\text { Pacientes } \\
\text { Seguidos }\end{array}$ & $\begin{array}{c}\text { Tempode } \\
\text { Cicatrização } \\
\text { (Dias) }\end{array}$ & Seguimento & Recidivas \\
\hline Al-Hassan e cols. ${ }^{5}$ & 1990 & Excisão - Método aberto & 50 & 42 & 91 & 25 meses & $12 \%$ \\
\hline Morell e cols. ${ }^{6}$ & 1991 & Excisão - Método aberto & 28 & 28 & 147 & $0-6$ anos & $3.5 \%$ \\
\hline Sondenaa e cols. ${ }^{7}$ & 1996 & Excisão - Método aberto & 60 & 59 & 85 & 4.2 anos & $5 \%$ \\
\hline Spivak e cols. ${ }^{2}$ & 1996 & Excisão - Método aberto & 47 & 47 & 56 & 2.6 anos & $13 \%$ \\
\hline Rosato e cols. ${ }^{4}$ & 1997 & Excisão - Método aberto & 48 & 48 & - & 16 meses & $8 \%$ \\
\hline Nahas e cols. ${ }^{8}$ & 1997 & Excisão - Método aberto & 39 & 39 & - & 5.3 anos & 0 \\
\hline Viciano et $a l^{9}$ & 2000 & Excisão - Método aberto & 38 & 38 & 68 & 74 meses & 0 \\
\hline Lee e cols. ${ }^{1}$ & 2000 & Excisão - Método aberto & 24 & 24 & 49 & 4 anos & $8.3 \%$ \\
\hline Kronborg e cols. ${ }^{10}$ & 1985 & Excisão - Método fechado & 33 & 29 & 14 & 3 anos & $25 \%$ \\
\hline Bissett e cols. ${ }^{11}$ & 1987 & Excisão - Método fechado & 57 & 40 & 47 & $5-8$ anos & $28.5 \%$ \\
\hline Al-Hassan e cols. ${ }^{5}$ & 1990 & Excisão - Método fechado & 46 & 45 & 10.3 & 40 meses & $20 \%$ \\
\hline Khaira e cols. ${ }^{3}$ & 1995 & Excisão - Método fechado & 46 & 40 & - & 23.5 meses & $17.5 \%$ \\
\hline Spivak e cols. $^{2}$ & 1996 & Excisão - Método fechado & 56 & 56 & 14 & 3.3 anos & $11 \%$ \\
\hline Sondenaa e cols. ${ }^{7}$ & 1996 & Excisão - Método fechado & 60 & 60 & - & 4.2 anos & $10 \%$ \\
\hline Rosato e cols. ${ }^{4}$ & 1997 & Excisão - Método fechado & 14 & 14 & - & 16 meses & $14 \%$ \\
\hline Lee e cols. ${ }^{1}$ & 2000 & Excisão - Método fechado & 10 & 10 & 40 & 4 anos & $10 \%$ \\
\hline Al-Jaberi ${ }^{12}$ & 2001 & Excisão - Método fechado & 46 & 46 & - & 3 anos & $4 \%$ \\
\hline Meban e cols. ${ }^{13}$ & 1982 & Marsupialização & 31 & 31 & 29 & 4 anos & $3.3 \%$ \\
\hline Vaula e cols. ${ }^{14}$ & 1986 & Marsupialização & 79 & 79 & $20-25$ & $0-6$ anos & $1.2 \%$ \\
\hline Spivak e cols. ${ }^{2}$ & 1996 & Marsupialização & 26 & 26 & 35 & 3.3 anos & $4 \%$ \\
\hline Lee e cols. ${ }^{1}$ & 2000 & Marsupialização & 4 & 4 & 49 & 4 anos & 0 \\
\hline Silva ${ }^{15}$ & 1972 & Incisão e curetagem & 80 & 64 & 27 & 39 meses & $1.25 \%$ \\
\hline Edwards ${ }^{16}$ & 1977 & Incisão e curetagem & 120 & 102 & 39 & 5 anos & $4.4 \%$ \\
\hline McLaren ${ }^{17}$ & 1984 & Incisão e curetagem & 18 & 18 & 42 & 20 anos & $10 \%$ \\
\hline Bissett e cols. ${ }^{11}$ & 1987 & Incisão e curetagem & 55 & 46 & 48 & $5-8$ anos & $19.5 \%$ \\
\hline Nahas e cols. ${ }^{8}$ & 1997 & Incisão e curetagem & 115 & 115 & - & 3.2 anos & $3.5 \%$ \\
\hline Mansoory e cols. ${ }^{18}$ & 1982 & Retalhos & 120 & 120 & $7-8$ & $1-9$ anos & $1.7 \%$ \\
\hline Guyuron e cols. ${ }^{19}$ & 1983 & Retalhos & 78 & 58 & 10 & $1-10$ anos & $5.1 \%$ \\
\hline Azab e cols. ${ }^{20}$ & 1984 & Retalhos & 30 & 30 & 10 & 3.5 anos & $3.3 \%$ \\
\hline Khatri e cols. ${ }^{21}$ & 1994 & Retalhos & 5 & 5 & - & $0.5-4.5$ anos & 0 \\
\hline Schoeller e cols. ${ }^{22}$ & 1997 & Retalhos & 24 & 24 & - & 4.5 anos & 0 \\
\hline Dylek e cols. ${ }^{23}$ & 1998 & Retalhos & 23 & 23 & - & 1.5 anos & 0 \\
\hline Milito e cols. ${ }^{24}$ & 1998 & Retalhos & 67 & 67 & - & 74.4 meses & 0 \\
\hline Bozkurt e cols. ${ }^{25}$ & 1998 & Retalhos & 24 & 24 & - & 27 meses & 0 \\
\hline Abu Galala e cols. ${ }^{26}$ & 1999 & Retalhos & 24 & 24 & - & 1.5 anos & 0 \\
\hline Lee e cols. ${ }^{1}$ & 2000 & Retalhos & 19 & 19 & 55 & 4 anos & 0 \\
\hline Bascom $^{27}$ & 1980 & Bascom & 50 & 44 & 21 & 2 anos & $8 \%$ \\
\hline Bascom $^{28}$ & 1983 & Bascom & 161 & 149 & 21 & 25 dias & $16 \%$ \\
\hline Mosquera e cols. ${ }^{29}$ & 1995 & Bascom & 41 & 41 & 39 & & $7.3 \%$ \\
\hline Senapati e cols. ${ }^{30}$ & 2000 & Bascom & 218 & 207 & - & 1 ano & $10 \%$ \\
\hline Mann e cols. ${ }^{31}$ & 1987 & Karidakys & 30 & 30 & 16 & 3 meses & $20 \%$ \\
\hline Karidakys ${ }^{32}$ & 1992 & Karidakys & 7471 & 6545 & 9 & $2-20$ anos & $1 \%$ \\
\hline Kitchen ${ }^{33}$ & 1996 & Karidakys & 141 & 114 & 10 & $2-20$ anos & $4 \%$ \\
\hline Anyanwu e cols. ${ }^{34}$ & 1998 & Karidakys & 28 & 24 & - & 3 anos & $4 \%$ \\
\hline
\end{tabular}


O tempo médio de cicatrização foi muito mais longo no grupo da excisão sem sutura primária (81,28 dias) do que nos demais grupos, mas este dado não foi considerado suficientemente confiável, porque diversos estudos não revelaram esta informação, principalmente os artigos do grupo dos retalhos. Devido a esta falta de informação na literatura, uma análise estatística comparando os tempos de cicatrização não foi realizada.

Em termos de taxa de recidiva, o grupo da excisão com sutura primária e o grupo da cirurgia de Bascom apresentaram resultados inferiores estatisticamente aos demais $(15,29 \%$, e $11,56 \%$ respectivamente), com p $<0,001$. O grupo da cirurgia de Karydakys mostrou as menores taxas de recidiva $(1,14 \%)$, porém de forma estatisticamente comparável aos procedimentos com retalhos $(1,53 \%)$, masrsupialização $(2,14 \%)$, incisão e curetagem $(6,08 \%)$ e excisão sem sutura primária $(6,15 \%)$ (Tabela 2)

\section{DISCUSSÃO}

O tratamento de escolha do cisto pilonidal deve apresentar uma taxa mínima de recidiva e um curto período de convalescença pós-operatória ${ }^{35}$. Normalmente, os primeiros sinais da doença ocorrem na fase aguda, sob a forma de um abscesso. O tratamento clássico nesta fase consiste basicamente em incisão e drenagem sob anestesia local, seguido por uma cicatrização da ferida por segunda intenção ${ }^{36}$. Porém, em muitos pacientes, a doença desenvolve um caráter recidivante.

Allen-Mersh propõe que como o cisto pilonidal é considerado uma doença adquirida, as recidivas tardias representam, na realidade, novos cistos adquiridos e não verdadeiras recidivas ${ }^{37}$. Nesta situação, um seguimento à longo prazo revelaria apenas o número crescente de pacientes com nova doença ${ }^{38}$. Além disso, é preciso considerar a distinção entre a cicatrização tardia e verdadeira recidiva. Esta última representa excisão incompleta do seio pilonidal na cirurgia inicial ou a formação de um novo foco da doença, enquanto que a cicatrização tardia também pode resultar na formação de tecido de granulação com pêlos ${ }^{3}$. A grande maioria dos autores tende a considerar o desenvolvimento de novos sintomas (abscessos recidivantes, drenagem mucóide ou purulenta crônica e dor) e a presença de pêlos e orifícios na região sacrococcígea como sendo uma recidiva da doença.

A excisão completa de todo cisto pilonidal é amplamente praticada. $\mathrm{O}$ orifício de abertura dos seios pode ser cateterizado, o trajeto ou trajetos são identificados e descobertos. Grandes incisões e excisões de tecidos normais devem ser evitadas. Ainda permanece controversa a conduta mais adequada a ser tomada em relação à ferida após a excisão. Ela deve ser deixada aberta ${ }^{5,7,39}$ ou ser fechada primariamente? ?,33,34 $^{3}$ Quando a ferida é fechada primariamente, os pacientes devem ser acompanhados de perto e deve-se estar alerta para infecções da ferida, seromas e/ou hematomas. Se a ferida é deixada aberta, o uso de soluções hidrocolóides pode diminuir a dor pós-operatória e aumentar o conforto do paciente ${ }^{9}$.

A técnica de marsupialização para o tratamento do cisto pilonidal foi descrita por Buie em 1937. Faz-se uma incisão na pele e os trajetos são amplamente abertos, porém deixando a base dos trajetos intacta. As pontas da pele são suturadas até a base, diminuindo o tamanho da ferida e também o tempo de cicatrização.

Há procedimentos cirúrgicos que mantém a ferida principal longe da linha média. Deste modo, elimina-se a tensão do tecido e facilita-se a cicatrização primária ${ }^{9}$. Karydakis, desde 1968, publicou diversos trabalhos com uma técnica de fechamento assimétrico, apresentando excelentes resulta$\operatorname{dos}^{32,40}$. Bascom, desde 1980, publicou artigos com os resultados das remoções dos folículos e drenagem lateral. Este procedimento pode ser realizado em ambulatório com mínima morbidade ${ }^{27,28}$.

A incisão e curetagem é uma modificação e provável aprimoramento da técnica inicial usada por Buie. São feitas apenas incisão e curetagem. É baseada em achados histológicos que mostram que o cisto pilonidal é basicamente um processo inflamatório granulomatoso causado pela presença de corpos estranhos no tecido subcutâneo, ou seja, pêlos, e que eles somente podem vir de fora já que não há folículos pilosos na parede do cisto. Esta técnica também pode ser utilizada em casos agudos da doença, alternativamente ao uso de drenagem apenas ${ }^{41}$.

A excisão do cisto pilonidal e o fechamento com retalhos reduz o tempo de cicatrização para duas semanas em média, em $90 \%$ dos pacientes, com uma taxa de recidiva similar à de outros procedimentos ${ }^{36}$. Entre estes retalhos há o

Tabela 2 - Meta-análise das taxas de recidiva.

\begin{tabular}{lccc}
\hline & Sem Recidivas & Com Recidivas & Total \\
\hline Excisão - método aberto & $305(93,85 \%)$ & $20(6,15 \%)$ & 325 \\
Excisão - método fechado & $288(84,71 \%)$ & $52(15,29 \%)$ & 340 \\
Marsupialização & $137(97,86 \%)$ & $3(2,14 \%)$ & 140 \\
Incisão e curetagem & $324(93,92 \%)$ & $21(6,08 \%)$ & 345 \\
Retalhos & $388(98,47 \%)$ & $6(1,53 \%)$ & 394 \\
Bascom & $390(88,43 \%)$ & $51(11,56 \%)$ & 441 \\
Karidakys & $6636(98,85 \%)$ & $77(1,14 \%)$ & 6713 \\
Total & 8468 & 230 & \\
\hline
\end{tabular}


fasciocutâneo em V-Y, a plastia em Z, a plastia em W, o rombóide e o glúteo máximo. Estes tipos de reconstrução permitem que o tecido seja aproximado sem tensão, e desta maneira, há redução do risco de ruptura da ferida. $\mathrm{O}$ fechamento sem tensão oferece benefícios adicionais, como redução da dor no pósoperatório, menor restrição de atividades e menor período de internação ${ }^{21}$. Porém, devido ao grande número de retalhos citados na literatura, um estudo comparativo entre todos deve ser realizado para que se possa definir qual o melhor em termos de tempo de cicatrização e taxas de recidiva. Atualmente, os retalhos são utilizados nos casos de recidiva da doença.

Este estudo teve por objetivo contemplar os principais procedimentos encontrados na literatura, embora muitos outros tenham sido descritos. Esta meta-análise mostrou que a maior parte dos métodos é comparável em termos de recidiva do cisto pilonidal. Apenas o procedimento de Bascom e a excisão com sutura primária foram estatisticamente inferiores aos demais métodos. Uma possível explicação reside no fato de que a maioria dos procedimentos remove o cisto pilonidal por completo, deixando riscos comparáveis de recidiva. Isto também explica parcialmente o motivo dos cirurgiões adotarem diferentes estratégias cirúrgicas mundialmente. Não há, portanto, um melhor tratamento, e a escolha é normalmente baseada na experiência pessoal de cada cirurgião.

O método de escolha de nossa equipe é a incisão e curetagem, porque, como relatado por Silva ${ }^{41}$, pode ser utilizado em todos os casos, mesmo em vigência de complicações, dispensa cuidados pré-operatórios especiais, é fácil de ser realizado, não demanda envolvimento da fáscia sacral ou periósteo, preserva o tecido normal, e causa uma ferida cirúrgica pequena. Há pouco desconforto pós-operatório, requer curto período de internação, tem cicatrização rápida e sem dor. Além disso, a curetagem pode ser realizada nos casos agudos da doença pilonidal, e considerando-se a alta incidência de sintomas crônicos tardios após a drenagem do abscesso, pode-se evitar a necessidade de uma segunda intervenção cirúrgica.

\begin{abstract}
Background: This study intends to analyse and compare several surgical procedures described for the treatment of pilonidal disease. Methods: Thirty-four published articles were selected, totalizing 8698 surgically treated patients. A meta-analysis was performed to compare seven main surgical techniques described in literature, in terms of recurrence rates and postoperative healing time. Results: From the total number of patients, 230 of them (2,6\%) presented with recurrences. The postoperative healing time was significantly longer in the group of excision without suture. The recurrence rate was statistically similar between the following techniques: excision without suture, marsupialization, incision and curettage, excision with flap and the Karidakys procedure. Procedures that had higher rates of recurrence (statistically significant $-p<0,001$ ) were the excision with primary suture and the Bascom procedure. Conclusion: This study concludes that the results in terms of recurrence rate are statistically similar between most procedures, with the exception of excision with primary suture and the Bascom procedure. The healing time was higher in the patients treated by excision without suture.
\end{abstract}

Key Words: Pilonidal cyst; Pilonidal disease; Marsupialization; Sacral abscess; Incision and curettage.

\section{REFERÊNCIAS}

1. Lee HC, Ho YH, Seow CF, et al. - Pilonidal disease in Singapore: clinical features and management. Aust N Z J Surg, 2000, 70(3):196-198

2. Spivak H, Brooks VL, Nussbaum M, et al. - Treatment of chronic pilonidal disease. Dis Colon Rectum, 1996, 39(10):11361139.

3. Khaira HS, Brown JH - Excision and primary suture of pilonidal sinus - Ann R Coll Surg Engl, 1995, 77: 242-244.

4. Rosato L, Fornero G, Luc AR, et al. - Il trattamento radicale della cisti pilonidale sacrococcígea. Minerva Chir, 1997, 52(10):1277-1279.

5. Al-Hassan HK, Francis IM, Neglen P - Primary closure or secondary granulation after excision of pilonidal sinus? Acta Chir Scand, 1990, 156(10):695-699.

6. Morell V, Charlton BL, Deshmukh N - Surgical treatment of pilonidal disease: comparison of three different methods in fiftynine cases. Mil Med, 1991,156(3):144-146.

7. Sondenaa K, Nesvik I, Andersen E, et al. - Recurrent pilonidal sinus after excision with closed or open treatment: final result of a randomised trial. Eur J Surg, 1996, 162(3): 237-240.

8. Nahas SC, Sobrado Jr CW, Araujo SEA, et al. - Resultados do tratamento cirúrgico de cisto pilonidal não-complicado. Rev Hosp Clin Fac Med Univ São Paulo, 1997, 52(6):287290.
9. Viciano V, Castera JE, Medrano J, et al. - Effect of hydrocolloid dressings on healing by second intention after excision of pilonidal sinus. Eur J Surg, 2000, 166(3):229-232.

10. Kronborg O, Christensen K, Zimmermann-Nilsen C - Chronic pilonidal disease: a randomized trial with a complete 3-year follow-up. Br J Surg, 1985, 72(4):303-304.

11. Bissett IP, Isbister WH - The management of patients with pilonidal disease - a comparative study. Aust N Z J Surg, 1987, 57(12):939-942.

12. Al-Jaberi TM - Excision and simple primary closure of chronic pilonidal sinus. Eur J Surg, 2001, 167(2):133-135.

13. Meban S, Hunter E - Outpatient treatment of pilonidal disease. Can Med Assoc J, 1982, 126(8):941.

14. Vaula JL, Badaro JA, Nacusse E, et al. - Enfermedad pilonidal sacrococcigea. Prensa Med Argent, 1986, 73(11): 489-491

15. Silva JH - Cisto pilonidal sacrococcígeo. Contribuição ao tratamento cirúrgico pela técnica da incisão e curetagem. Dissertação. São Paulo. Faculdade de Medicina da Universidade de São Paulo, 1972.

16. Edwards MH - Pilonidal sinus: a 5-year appraisal of the MillarLord treatment. Br J Surg, 1977, 64(12):867-868.

17. McLaren CA - Partial closure and other techniques in pilonidal surgery: an assessment of 157 cases. Br J Surg, 1984, 71(7): 561-562.

18. Mansoory A, Dickson D - Z-plasty for treatment of disease of pilonidal sinus. Surg Gynecol Obstet, 1982, 155(3): 409-411. 
19. Guyuron B, Dinner MI, Dowden RV - Excision and grafting in treatment of recurrent pilonidal sinus disease. Surg Gynecol Obstet, 1983, 156(2): 201-204.

20. Azab AS, Kamal MS, Saad RA, et al. - Radical cure of pilonidal sinus by a transposition rhomboid flap. Br J Surg, 1984, 71(2): 154-155.

21. Khatri VP, Espinosa MH, Amin AK - Management of recurrent pilonidal sinus by simple V-Y fasciocutaneous flap. Dis Colon Rectum, 1994, 37(12):1232-1235.

22. Schoeller T, Wechselberger G, Otto A, et al. - Definite surgical treatment of complicated recurrent pilonidal disease with a modified fasciocutaneous V-Y advancement flap. Surgery, 1997, 121(3):258-263.

23. Dylek ON, Bekereciodlu M - Role of simple V-Y advancement flap in the treatment of complicated pilonidal sinus. Eur J Surg, 1998, 164(12):961-964.

24. Milito G, Cortese F, Casciani CU - Rhomboid flap procedure for pilonidal sinus: results from 67 cases. Int J Colorectal Dis, 1998, 13(3):113-115.

25. Bozkurt MK, Tezel E - Management of pilonidal sinus with the Limberg flap. Dis Colon Rectum, 1998, 41(6):775-777.

26. Abu Galala KH, Salam IM, Abu Samaan KR, et al. - Treatment of pilonidal sinus by primary closure with a transposed rhomboid flap compared with deep suturing: a prospective randomized clinical trial. Eur J Surg, 1999, 165(5): 468-472.

27. Bascom $\mathrm{J}$ - Pilonidal disease: origin from follicles of hairs and results of follicle removal as treatment. Surgery, 1980, 87(5):567572.

28. Bascom J - Pilonidal disease: Long-term results of follicle removal. Dis Colon Rectum, 1983, 26(12):800-807.

29. Mosquera DA, Quayle JB - Bascom's operation for pilonidal sinus. J R Soc Med, 1995, 88(1):45P-46P.

30. Senapati A, Cripps NP, Thompson MR - Bascom's operation in the day-surgical management of symptomatic pilonidal sinus. Br J Surg, 2000, 87(8):1067-1070.
31. Mann CV, Springall R. - "D” excision for sacrococcygeal pilonidal sinus disease. J R Soc Med, 1987, 80(5):292-295.

32. Karydakis GE - Easy and successful treatment of pilonidal sinus after explanation of its causative process. Aust N Z J Surg, 1992, 62(5):385-389.

33. Kitchen PR - Pilonidal sinus: experience with the Karydakis flap. Br J Surg, 1996, 83(10):1452-1455.

34. Anyanwu AC, Hossain S, Williams A, et al. - Karydakis operation for sacrococcygeal pilonidal sinus disease: experience in a district general hospital. Ann R Coll Surg Engl, 1998, 80(3):197-199.

35. Matter I, Kunin J, Schein M, et al. - Total excision versus nonresectional methods in the treatment of acute and chronic pilonidal disease. Br J Surg, 1995, 82(6):752-753.

36. Quinodoz PD, Chilcott M, Grolleau JL, et al. - Surgical treatment of sacrococcygeal pilonidal sinus disease by excision and skin flaps: the Tolouse experience. Eur J Surg, 1999, 165(11):10611065.

37. Allen-Mersh TG - Pilonidal sinus: finding the right track for treatment. Br J Surg, 1990, 77(2):123-132.

38. Isbister WH, Prasad J - Pilonidal disease. Aust N Z J Surg, 1995, 65(8):561-563.

39. Fuzun M, Bakir H, Soylu M, et al - Which technique for treatment of pilonidal sinus - open or closed? Dis Colon Rectum, 1994, 37(11):1148-1150.

40. Karydakis GE - New approach to the problem of pilonidal sinus. Lancet, 1973, 2(7843):1414-1415.

41. da Silva JH - Pilonidal cyst: cause and treatment. Dis Colon Rectum, 2000, 43(8): 1146-1156.

Endereço para correspondência:

Victor Strassmann

Rua S. Domingos Savio, 137 / WS81 - Vila Ida

CEP: 05455-040 - São Paulo - SP

e-mail: vstrass@uol.com.br 